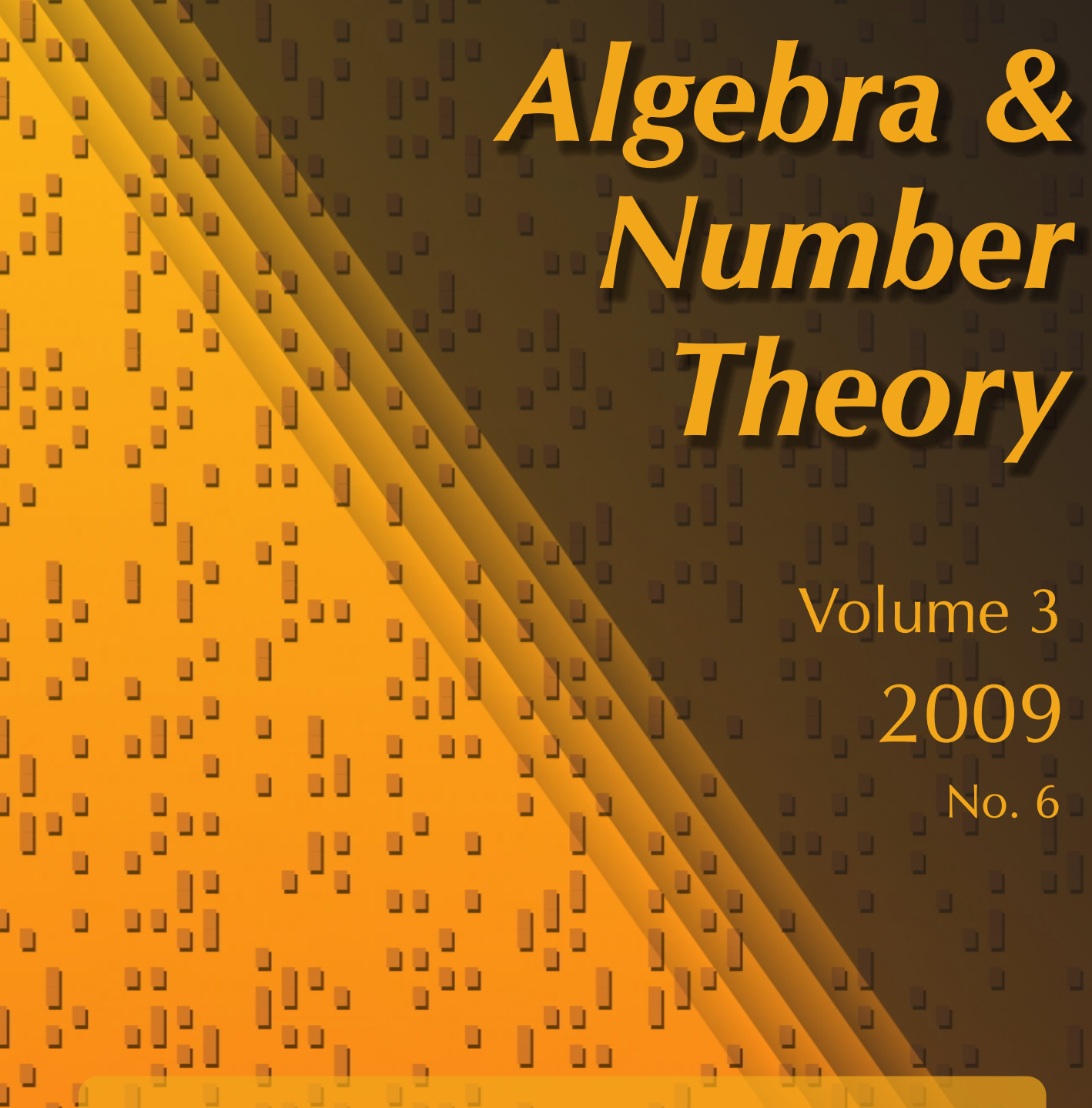

A pencil of Enriques surfaces of index one with no section

\lrcorner$\lrcorner \quad$ Jason Michael Starr

\lrcorner

」

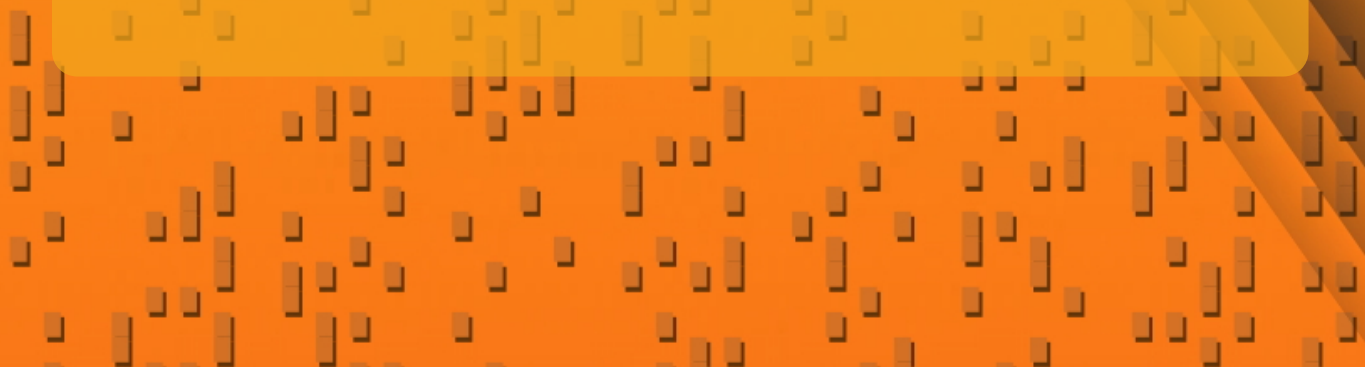




\title{
A pencil of Enriques surfaces of index one with no section
}

\author{
Jason Michael Starr
}

\begin{abstract}
Monodromy arguments and deformation-and-specialization are used to prove existence of a pencil of Enriques surfaces with no section and index 1. The same technique "completes" the strategy from Graber et al. (2005) proving that the family of witness curves for dimension $d$ depends on the integer $d$.
\end{abstract}

\section{Introduction}

This paper uses monodromy and deformation-and-specialization to answer some questions related to [Graber et al. 2005].

Theorem 1.1. There exists a positive integer $t$ (which the reader can readily compute) such that the following holds. Let $k$ be any algebraically closed field with $\operatorname{char}(k) \neq 2,3$ whose transcendence degree over the prime field is $\geq t$. This holds, for instance, if $k$ is uncountable. There exists a flat, projective $k$-morphism $\pi: \mathscr{X} \rightarrow \mathbb{P}_{k}^{1}$ with the following properties.

(i) The geometric generic fiber of $\pi$ is a smooth Enriques surface.

(ii) The invertible sheaf $\pi_{*}\left[\omega_{\pi}^{\otimes 2}\right]$ has degree 6 .

(iii) For the function field $K$ of $\mathbb{P}_{k}^{1}$ and the generic fiber $X_{K}$ of $\pi$, the residue field of each closed point of $X_{K}$ has degree $\geq 3$ as an extension of $K$. Moreover, there exists at least one closed point of degree 3 and at least one closed point of degree 4. So the greatest common divisor of all degrees of closed points equals 1 .

Moreover every "very general" Enriques surface over $k$ is a fiber of such a family.

What is the relevance of this result? First of all, in [Graber et al. 2005, Corollary 1.4] it was proved that there exists an Enriques surface $X$ defined over the function field $K=\mathbb{C}(B)$ of some complex curve $B$ such that $X$ has no $K$-rational points.

MSC2000: primary 14G05; secondary 14D06.

Keywords: rational point, Enriques surface.

Partially supported by NSF Grants DMS-0201423, DMS-0353692, DMS-0553921, DMS-0758521, and an Arthur P. Sloan fellowship. 
This answered a question which Serre asked [Colmez and Serre 2001, page 153]. Next, shortly after the results of [Graber et al. 2005] were proved, Lafon [2004] even gave an explicit pencil of Enriques surfaces defined over $(\mathbb{Z}[1 / 2])[t][1 / f(t)]$ for an explicit monic polynomial $f(t)$ such that for every field $k$ of characteristic $\neq 2$, the base change of the Enriques surface to $K=k(t)$ has no $K$-rational point.

Motivated by this, Hélène Esnault posed to Joe Harris the important question of whether or not these examples can be somehow explained "cohomologically". In particular, she posited that the Enriques surfaces from [Graber et al. 2005] have the stronger property that every 0 -cycle has degree divisible by 4 . Similarly, for Lafon's Enriques surface, every 0-cycle has even degree. This suggests that these examples, and by extension, perhaps all examples, can be understood in terms of obstructions which are defined as elements in Galois cohomology groups of the fraction field $K$ of the base $B$. These obstructions are compatible with restriction and corestriction for finite, separable field extensions $L / K$. In concrete terms what this implies is that the order of the obstruction (as an element in the appropriate torsion Galois cohomology group) divides the degree over $K$ of the residue field $L$ of every closed point of $X$ (for which $L / K$ is separable). Thus, if one of these obstructions is nonzero, then the greatest common divisor of the degrees of all (separable) closed points is $>1$. Therefore, since the greatest common divisor of all (separable) closed points is $>1$ in the Enriques surfaces of [Graber et al. 2005] and [Lafon 2004], perhaps there is a cohomological explanation for those Enriques surfaces.

In Theorem 1.1, the degree 3 and degree 4 points are separable (since the characteristic is not 2,3$)$, and $\operatorname{gcd}(3,4)$ equals 1 . Therefore nonexistence of rational points is not due to a cohomological obstruction as above. Of course there may be a cohomological obstruction defined in a different way which does "explain" the nonexistence of rational points of these varieties.

For comparison, note that the greatest common divisor of degrees of closed points in Lafon's example equals 2. Although I am not certain, I expect that also in the examples from [Graber et al. 2005] (which are defined only in a very indirect manner), the greatest common divisor is again $>1$.

Proposition 1.2. Let $k$ be an algebraically closed field having infinite transcendence degree over its prime subfield, for example, an uncountable algebraically closed field. Let $B$ be an integral, normal, projective $k$-scheme of dimension $\geq 2$. Let $M$ be an integral quasiprojective $k$-variety. And let $\mathscr{C} \subset M \times_{k} B$ be a closed subscheme which is flat of relative dimension 1 over $M$ with irreducible geometric fibers and which dominates $B$, that is, $(M, \mathscr{C})$ is a family $\left(\mathscr{C}_{m}\right)_{m \in M}$ of irreducible curves $\mathscr{C}_{m}$ in $B$ such that $\cup_{m \in M} \mathscr{C}_{m}$ contains a dense open subset of $B$. For all integers $n$ which are sufficiently positive, there exists a projective, dominant morphism of integral $k$-schemes $\pi: \mathscr{X} \rightarrow B$ having relative dimension $n$ and whose 
restriction to each geometric fiber of $\mathscr{b} \rightarrow M$ has a section, but whose restriction to some smooth curve in B has no section. In the language of [Graber et al. 2005], this means that for every family of curves $(M, \mathscr{C})$, for all sufficiently positive integers $n$, the family is not a witness family for relative dimension $n$.

This proposition completes the sketch given in [Graber et al. 2005, Section 7.3]. Just to repeat, the significance is that there does not exist a family of curves in $B$ which is simultaneously for all integers $n$ a witness family for relative dimension $n$.

In proving Theorem 1.1 and Proposition 1.2 it will be useful to recall the definition of the index.

Definition 1.3. Let $X$ be a finite type scheme, algebraic space, algebraic stack, etc. over a field $K$. The index and the minimal degree are,

$$
\begin{gathered}
I(K, X)=\operatorname{gcd}\{[L: K] \mid X(L) \neq \varnothing\}, \\
M(K, X)=\min \{[L: K] \mid X(L) \neq \varnothing\} .
\end{gathered}
$$

The proofs of Theorem 1.1 and Proposition 1.2 both use the same technique; here is a brief description for Theorem 1.1. Over $\mathbb{P}^{1}$ a family of reducible surfaces is given whose monodromy group acts as the full group of symmetries of the dual graph of the geometric generic fiber. This dual graph is the 2-skeleton of a cube. There is an action of $\mathbb{Z} / 2 \mathbb{Z}$ acting fiberwise on the family. The quotient by this action is a pencil $\mathscr{X} / \mathbb{P}^{1}$ of degenerate Enriques surfaces, that is, the geometric generic fiber deforms to a smooth Enriques surface. The 8 vertices of the cube give a degree 4 multisection of the pencil. The 6 faces of the cube give a degree 3 multisection of the pencil. By monodromy considerations every multisection of $X$ has degree $\geq 3$. The pencil $X$ together with the degree 3 and degree 4 multisections deforms to a pencil of surfaces whose geometric generic fiber is a smooth Enriques surface. For such a deformation which is sufficiently general, $M\left(K, X_{K}\right)$ equals 3 and $I\left(K, X_{K}\right)$ equals 1 .

\section{The construction for hypersurfaces}

Let $n-1$ be a positive integer. The goal of this section is to construct the morphism $\pi$ of relative dimension $n-1$ satisfying the condition from Proposition 1.2. The morphism is constructed as a family of hypersurfaces in $\mathbb{P}^{n}$ of degree $d>n$ parameterized by $B$, that is, $\mathscr{X}$ will be constructed as a closed subscheme of $B \times{ }_{k} \mathbb{P}_{k}^{n}$ whose fibers over $B$, considered as closed subschemes of $\mathbb{P}^{n}$, are hypersurfaces of degree $d$. Of course this is equivalent to giving a rational transformation from $B$ to the Hilbert scheme $\operatorname{Hilb}_{\mathbb{P}_{k}^{n} / k}^{P_{d}(t)}$ of degree $d$ hypersurfaces in $\mathbb{P}^{n}$ (where $P_{d}(t)$ is the appropriate Hilbert polynomial). This Hilbert scheme is itself a projective space $\mathbb{P}_{k}^{N_{d, n}}$, where $N_{d, n}$ equals $\left(\begin{array}{c}n+d \\ n\end{array}\right)-1$. The goal of this section is to construct 
1-parameter families of degree $d$ hypersurfaces in $\mathbb{P}^{n}$ with no section, and then explain why some smooth curves in $B$ give such 1-parameter families.

The parameter space for degree d hypersurfaces. Let $k$ be a field. For simplicity assume that $k$ is algebraically closed. Let $d, n>0$ be integers. Let $V$ be a $k$-vector space of dimension $n+1$ so that $\mathbb{P}(V)$ is isomorphic to $\mathbb{P}_{k}^{n}$. For keeping track of the dense notation, it is simpler to use $\mathbb{P}(V)$ throughout rather than $\mathbb{P}_{k}^{n}$. There seem to be different conventions as to the meaning of $\mathbb{P}(V)$. Thus to make precise, $\mathbb{P}(V)$ is defined here to be

$$
\mathbb{P}(V):=\operatorname{Proj} \underset{i \geq 0}{\bigoplus} \operatorname{Sym}_{k}^{i}\left(V^{\vee}\right) .
$$

In particular, there exists an invertible sheaf $\mathcal{O}_{\mathbb{P}(V)}(-1)$ and a monomorphism of coherent sheaves,

$$
u: \mathbb{O}_{\mathbb{P}(V)}(-1) \rightarrow V \otimes_{k} \mathcal{O}_{\mathbb{P}(V)}
$$

whose restriction over every sufficiently small Zariski open subset of $\mathbb{P}(V)$ is a split monomorphism. Moreover, the triple $\left(\mathbb{P}(V), \mathbb{O}_{\mathbb{P}(V)}(-1), u\right)$ is universal among all such triples of a $k$-scheme, an invertible sheaf, and a locally split monomorphism of coherent sheaves. This is the universal property of projective space used here. (Of course, by taking adjoint maps, it is equivalent to the property appearing elsewhere which uses locally split epimorphisms to an invertible sheaf.)

There is a minor positive characteristic issue that merits some discussion. The symmetric product $\operatorname{Sym}^{d}\left(V^{\vee}\right)$ is the maximal $k$-vector space quotient of $\bigotimes_{k}^{d}\left(V^{\vee}\right)$, which is invariant for the natural $k$-linear action of the symmetric group $\mathfrak{S}_{d}$, that is, $\operatorname{Sym}^{d}\left(V^{\vee}\right)$ is the space of $\mathfrak{S}_{d}$-coinvariants. In particular, $\bigoplus_{i \geq 0} \operatorname{Sym}^{i}\left(V^{\vee}\right)$ is the ring of polynomial functions from $V$ to $k$. The adjoint of the coinvariant quotient map gives a canonical $k$-vector space isomorphism between the dual $k$-vector space of $\operatorname{Sym}^{d}\left(V^{\vee}\right)$ and the subspace $\operatorname{Syt}^{d}(V)$ of $\mathfrak{S}_{d}$-invariants in $\bigotimes_{k}^{d}\left(V^{\vee}\right)$, that is,

$$
\operatorname{Hom}_{k}\left(\operatorname{Sym}^{d}\left(V^{\vee}\right), k\right)=\operatorname{Syt}^{d}(V) .
$$

Of course if $\operatorname{char}(k)$ is $>d$, then the induced map from invariants to coinvariants,

$$
\operatorname{Syt}^{d}(V) \rightarrow \operatorname{Sym}^{m}(V),
$$

is an isomorphism of $k$-vector spaces. But since Proposition 1.2 involves integers $d$ which are arbitrarily large, it is important to distinguish between the invariants and coinvariants.

Degree $d$ hypersurfaces in $\mathbb{P}(V)$ are parametrized by the projective space,

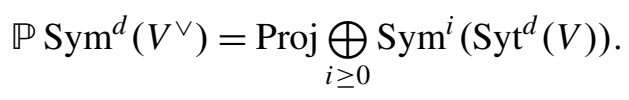


To be precise, there is a closed subscheme $\mathscr{Y} \subset \mathbb{P} \operatorname{Sym}^{d}\left(V^{\vee}\right) \times \mathbb{P}(V)$ such that the projection to $\mathbb{P} \operatorname{Sym}^{d}\left(V^{\vee}\right)$ is flat with geometric fibers being degree $d$ hypersurfaces in $\mathbb{P}(V)$. And the pair $\left(\mathbb{P} \operatorname{Sym}^{d}\left(V^{\vee}\right)\right.$, y) represents the Hilbert functor of $\mathbb{P}(V)$ for the appropriate Hilbert polynomial $P_{d, n}(t)$ of degree $d$ hypersurfaces, that is, for

$$
P_{d, n}(t)=\left(\begin{array}{c}
n+t \\
n
\end{array}\right)-\left(\begin{array}{c}
n+t-d \\
n
\end{array}\right) .
$$

Covers of $\mathbb{P}^{\mathbf{1}}$ by $\mathbb{P}^{\mathbf{1}}$. As an intermediate step, we will construct pencils of unions of $d$ hyperplanes. To be a little more precise, for a parameter curve $B$ (which we will assume to be $\mathbb{P}^{1}$ for simplicity), a pencil of unions of $d$ hyperplanes over $B$ is a closed subscheme $\mathscr{L} \subset B \times{ }_{k} \mathbb{P}(V)$ such that the projection $\mathscr{L} \rightarrow B$ is flat and the geometric generic fiber is a union of $d$ hyperplanes in $\mathbb{P}(V)$. Consider the normalization $(\mathscr{L})_{\text {nor }}$ of $\mathscr{L}$. The Stein factorization of the associated morphism $(\mathscr{Z})_{\text {nor }} \rightarrow B$ is a finite morphism $C \rightarrow B$. To construct the closed subscheme $\mathscr{L} \subset B \times \times_{k} \mathbb{P}(V)$, we will first construct the morphism $C \rightarrow B$. For simplicity, we will construct it so that both $B$ and $C$ are smooth, proper, connected curves of genus 0 . Thus, let $B$ and $C$ be given $k$-curves isomorphic to $\mathbb{P}_{k}^{1}$.

Lemma 2.1. For every integer $d \geq 2$, there exists a degree $d$, separably-generated $k$-morphism $f: C \rightarrow B$ such that $\operatorname{Gal}(k(C) / k(B))$ is the full symmetric group $\mathfrak{S}_{d}$.

Proof. One can prove this in many ways. The following proof is simple, but is only valid when $\operatorname{char}(k)$ is not 2 . The proofs I know for characteristic 2 are considerably longer. One such proof constructs the cover $f$ as a general deformation of a degree $d$, finite, flat, local complete intersection morphism $C \rightarrow B$ of nodal curves of genus 0 such that every component $C_{i}$ of $C$ maps to its image $B_{j}$ in $B$ as either an isomorphism or as a degree 2 cover,

$$
\mathbb{P}^{1} \rightarrow \mathbb{P}^{1}, \quad\left[X_{0}, X_{1}\right] \mapsto\left[X_{0}^{2}, X_{1}^{2}-X_{0} X_{1}\right],
$$

where the ramification points, resp. branch points, are smooth points of $C$, resp. $B$, that is, the total map is not ramified at the nodes of $C$. In characteristic 2, notice that the map above is an Artin-Schreier map. By analyzing the combinatorics of the associated set map from the dual graph of $C$ to the dual graph of $B$ "decorated" by the information of the degree of $C_{i} \rightarrow B_{j}$, one can show that for a general such morphism, every sufficiently general deformation is a morphism as in the lemma. So the result reduces to a small amount of combinatorial analysis. On the other hand, the following argument involves no combinatorial analysis.

Choose an isomorphism of $C$ with $\mathbb{P}^{1}$ and let $v_{d}: C \rightarrow \mathbb{P}_{k}^{d}$ be the associated $d$-uple Veronese morphism. Let $B$ be a general line in the dual projective plane, $B \subset\left(\mathbb{P}_{k}^{d}\right)^{\vee}$. Let $\Gamma \subset C \times{ }_{k} B$ be the corresponding pencil of degree $d$ divisors in $C$ parameterized by $B$. By [Deligne and Katz 1973, Théorème XVII.2.5], this is a 
Lefschetz pencil. Thus the base locus is transverse to $C$. Since $C$ is 1-dimensional, this means the base locus is empty. Therefore the projection $\Gamma \rightarrow C$ is an isomorphism. In other words, $\Gamma$ equals the graph of a morphism $f: C \rightarrow B$, that is, $\Gamma=\Gamma_{f}$. Since $\Gamma$ is a pencil of degree $d$ divisors in $C$, the morphism $f$ has degree $d$. Since this is a Lefschetz pencil, every ramification point $c_{i}$ of $f$ is an ordinary double point and the images $b_{i}=f\left(c_{i}\right)$ are all distinct points of $B$. Since char $(k)$ is not 2, this means that $f$ is a tamely ramified cover. By Riemann-Hurwitz for tamely ramified morphisms, there are $2 d-2$ branch points $b_{1}, \ldots, b_{2 d-2}$.

Denote $U=B \backslash\left\{b_{1}, \ldots, b_{2 d-2}\right\}$. Denote by $\bar{u}$ a geometric point of $U$. The morphism $f: f^{-1}(U) \rightarrow U$ is a finite étale morphism of degree $d$. Therefore it defines a group homomorphism from the tamely ramified fundamental group

$$
\phi: \pi_{1}^{t}(U, \bar{u}) \rightarrow \mathfrak{S}_{d},
$$

which is well-defined up to inner automorphism of $\mathfrak{S}_{d}$. By the description of the tamely ramified fundamental group in [Grothendieck 1962, Corollaire XIII.2.12], $\pi_{1}^{t}(U, \bar{u})$ is topologically generated by elements $\sigma_{1}, \ldots, \sigma_{2 d-2}$ such that the image of $\sigma_{i}$ is a topological generator of the inertia group at $b_{i}$. Because $f$ is simply ramified over $b_{i}$, the image $\phi\left(\sigma_{i}\right)$ is a transposition in $\mathfrak{S}_{d}$. Since $f^{-1}(U)$ is irreducible, Image $(\phi)$ is a transitive subgroup of $\mathfrak{S}_{d}$. Since $\operatorname{Image}(\phi)$ is a transitive subgroup of $\mathfrak{S}_{d}$ which is generated by transpositions, Image $(\phi)$ equals all of $\mathfrak{S}_{d}$. Thus the Galois group of $k(C) / k(B)$ is the full symmetric group $\mathfrak{S}_{d}$.

Norm sheaves and norm maps. Given a degree $d$ cover $f: C \rightarrow B$ as in Lemma 2.1, and given a $k$-morphism from $g: C \rightarrow \mathbb{P}\left(V^{\vee}\right)$ from $C$ to $\mathbb{P}\left(V^{\vee}\right)$, the parameter space of hyperplanes in $\mathbb{P}(V)$, there is an associated $k$-morphism from $B$ to $\mathbb{P}\left(\operatorname{Sym}^{d}\left(V^{\vee}\right)\right)$ sending a geometric point $b$ of $B$ to the reducible hypersurface which is the union over the $d$ points $c$ of $f^{-1}(b)$ of the corresponding hyperplane. This is made precise using norm sheaves and norm maps.

For simplicity, let $g: C \rightarrow \mathbb{P}\left(V^{\vee}\right)$ be a closed immersion whose image is a rational normal curve of degree $n$, for example,

$$
\mathbb{P}^{1} \rightarrow \mathbb{P}^{n}, \quad\left[X_{0}, X_{1}\right] \mapsto\left[X_{0}^{n}, X_{0}^{n-1} X_{1}, \ldots, X_{0}^{n-i} X_{1}^{i}, \ldots, X_{0} X_{1}^{n-1}, X_{1}^{n}\right] .
$$

Consider the pullback under $g$ of the tautological surjection, $V \otimes_{k} \mathscr{O}_{C} \rightarrow g^{*} \mathcal{O}(1)$. Of course $V \otimes_{k} O_{C}$ is canonically isomorphic to $f^{*}\left(V \otimes_{k} O_{B}\right)$. By adjointness of $f^{*}$ and $f_{*}$, there is an associated morphism of $\mathrm{O}_{B}$-modules,

$$
\beta: V \otimes_{k} O_{B} \rightarrow f_{*}\left(g^{*} \mathcal{O}(1)\right) .
$$

Now for every locally free $\mathbb{O}_{C}$-module $\mathscr{E}$ there is the associated norm sheaf on $B$, defined as

$$
\operatorname{Nm}_{f}(\mathscr{E})=\operatorname{Hom}_{\mathscr{O}_{B}}\left(\bigwedge^{d}\left(f_{*} \mathscr{O}_{C}\right), \bigwedge^{d}\left(f_{*}^{\mathscr{E}}\right)\right)
$$


There is also the associated norm map $\alpha_{\mathscr{E}}^{\prime}: \stackrel{d}{\otimes}\left(f_{*} \mathscr{E}\right) \rightarrow \mathrm{Nm}_{f}(\mathscr{E})$ of $\mathrm{O}_{B}$-modules, defined by

$$
e_{1} \otimes \cdots \otimes e_{d} \mapsto\left(c_{1} \wedge \cdots \wedge c_{d} \mapsto\left(c_{1} \cdot e_{1}\right) \wedge \cdots \wedge\left(c_{d} \cdot e_{d}\right)\right),
$$

for $e_{1} \otimes \cdots \otimes e_{d} \in \bigotimes^{d}\left(f_{*} \mathscr{E}\right)$ and $c_{1} \wedge \cdots \wedge c_{d} \in \wedge^{d}\left(f_{*} \mathcal{O}_{B}\right)$. In fact we will only need the restriction to the subsheaf of symmetric tensors, which we denote as follows

$$
\alpha_{\mathscr{E}}: \operatorname{Syt}^{d}\left(f_{*}^{\mathscr{C}}\right) \rightarrow \operatorname{Nm}_{f}(\mathscr{E}) .
$$

In particular, observe that $\operatorname{Nm}_{f}\left(\mathscr{O}_{C}\right)$ equals $\mathscr{O}_{B}$ and that for every local section $b$ of $f_{*} \mathrm{O}_{C}$, the norm $\alpha_{\mathscr{O}_{C}}(b \otimes \cdots \otimes b) \in \mathbb{O}_{B}$ is the usual norm of $b$.

The family of unions of hyperplanes. Denote by $\gamma$ the composition,

$$
\operatorname{Syt}^{d}(V) \otimes_{k} O_{B} \stackrel{\operatorname{Syt}^{d}(\beta)}{\longrightarrow} \operatorname{Syt}^{d}\left(f_{*} g^{*} O(1)\right) \stackrel{\alpha_{g^{*} \odot(1)}}{\longrightarrow} \operatorname{Nm}_{f}\left(g^{*} O(1)\right) .
$$

Because $\beta$ is surjective, also $\gamma$ is surjective. Because $f$ is finite, there exists a Zariski open covering $\left\{U_{i}\right\}$ of $B$ such that $g^{*} \mathcal{O}(1)$ is trivial on each open $f^{-1}\left(U_{i}\right)$. Thus

$$
\mathrm{Nm}_{f}\left(g^{*} \mathrm{O}(1)\right)
$$

is locally isomorphic to $\operatorname{Nm}_{f}\left(\mathscr{O}_{C}\right)$, that is, to $\mathscr{O}_{B}$. $\operatorname{So~Nm}_{f}\left(g^{*} \mathbb{O}(1)\right)$ is an invertible $\mathrm{O}_{B}$-module and $\gamma$ is locally a split epimorphism. Therefore, by the universal property of projective space, there is an induced morphism $h: B \rightarrow \mathbb{P} \operatorname{Sym}^{d}\left(V^{\vee}\right)$. Let $b$ be a geometric point of $B$ whose fiber $f^{-1}(b)$ is a reduced set $\left\{c_{1}, \ldots, c_{d}\right\}$. For every $i=1, \ldots, d$, the image $g\left(c_{i}\right)$ equals $\left[L_{i}\right]$ for a linear functional $L_{i} \in V^{\vee}$. And then $h(b)$ equals $\left[L_{1} \times \cdots \times L_{d}\right]$. The degree of $\mathrm{Nm}_{f}\left(g^{*} \mathcal{O}(1)\right)$ equals $n$. Thus $h^{*} \mathcal{O}(1)$ is an invertible $\mathbb{O}_{B}$-module of degree $n$.

Denote by $\mathscr{Y}_{h} \subset B \times \mathbb{P}(V)$ the preimage under $(h, \mathrm{Id})$ of the universal hypersurface 9 in $\mathbb{P} \operatorname{Sym}^{d}\left(V^{\vee}\right) \times \mathbb{P}(V)$. And denote by $\pi: \mathscr{Y}_{h} \rightarrow B$ the projection. Let $m=\min (d, n)$ and let $S_{d, n} \subset \mathbb{Z}_{\geq 0}$ denote the additive semigroup generated by $\left(\begin{array}{c}d \\ i\end{array}\right)$ for $i=1, \ldots, m$. Denote $k(B)$ by $K$ and denote by $\mathscr{Y}_{h, K}$ the generic fiber of $\pi$.

Proposition 2.2. For every irreducible multisection of $\pi$, there exists an integer $i=1, \ldots, m$ such that the degree of the multisection is divisible by $\left(\begin{array}{c}d \\ i\end{array}\right)$. The degree of every multisection is in the semigroup $S_{d, n}$. In particular, if $d$ is greater than $n$ then $M\left(K, \mathscr{Y}_{h, K}\right)$ equals $d$ and $I\left(K, \mathscr{Y}_{h, K}\right)$ is divisible by $\operatorname{gcd}\left(d,\left(\begin{array}{l}d \\ 2\end{array}\right), \ldots,\left(\begin{array}{l}d \\ n\end{array}\right)\right)$.

Proof. Denote by $U \subset B$ the largest open subset over which $f$ is étale and define $W=f^{-1}(U)$. For each $i=1, \ldots, m$, denote by $W_{i} / U$ the relative Hilbert scheme $\operatorname{Hilb}_{W / U}^{i}$. Because $W$ is étale over $U$, the fiber of $f$ over a geometric point $b$ of $B$ is a set of $d$ distinct points, $f^{-1}(b)=\left\{c_{1}, \ldots, c_{d}\right\}$, and the fiber of $\operatorname{Hilb}_{W / U}^{i}$ is the set of subsets of $f^{-1}(b)$ of size $i$. Every geometric fiber of $\mathscr{y}_{h} \times{ }_{B} U \rightarrow U$ is 
a union of $d$ hyperplanes. Denote by

$$
y_{h} \times{ }_{B} U=\mathscr{y}_{h}^{1} \sqcup \mathscr{Y}_{h}^{2} \sqcup \cdots \sqcup \mathscr{Y}_{h}^{n}
$$

the locally closed stratification where $\mathscr{y}_{h}^{i}$ is the set of points $x$ in precisely $i$ irreducible components of the geometric fiber $\mathscr{Y}_{h} \otimes_{\mathfrak{O}_{B}} \bar{\kappa}(\pi(x))$.

Because every finite subset of distinct closed points on a rational normal curve over an algebraically closed field is in linearly general position, $y_{h}^{i}$ is empty for all $i>m$. In particular every geometric fiber of $\mathscr{Y}_{h} \times{ }_{B} U \rightarrow U$ is a simple normal crossings variety. For each $i=1, \ldots, m$ the morphism $\mathscr{Y}_{h}^{i} \rightarrow U$ factors as an $\mathbb{A}^{n-i}$-bundle over $W_{i}$ over $U$.

For each irreducible multisection of $\pi$, there exists an integer $i \leq m$ (depending on the multisection) such that the generic point of the multisection is contained in $\mathscr{Y}_{h}^{i}$. Because $\operatorname{Gal}(k(C) / k(B))$ is the full symmetric group $\mathfrak{S}_{d}$, in particular it is $i$-transitive. Thus $W_{i}$ is irreducible. Therefore the degree of the multisection is divisible by $\operatorname{deg}\left(k\left(W_{i}\right) / k(U)\right)$ which equals $\left(\begin{array}{c}d \\ i\end{array}\right)$. So the degree of every multisection, irreducible or not, is in $S_{d, n}$. In particular, the degree is $\geq d$, that is, $M\left(K, \mathscr{Y}_{h, K}\right) \geq d$. Conversely, the intersection of $\mathscr{X}_{h, K}$ with a general line in $\mathbb{P}\left(V \otimes_{k} K\right)$ is a degree $d$ multisection. Therefore $M\left(K, \mathscr{Y}_{h, K}\right)$ equals $d$.

Denote by $\operatorname{Hom}\left(B, \mathbb{P} \operatorname{Sym}^{d}\left(V^{\vee}\right)\right)$ the Hom scheme [Grothendieck 1961, 4.c, p. 19]. And denote by $H_{n} \subset \operatorname{Hom}\left(B, \mathbb{P} \operatorname{Sym}^{d}\left(V^{\vee}\right)\right)$ the irreducible component parameterizing those morphisms of degree $n$. Denote the universal morphism by

$$
\chi: H_{n} \times_{k} B \rightarrow \mathbb{P} \operatorname{Sym}^{d}\left(V^{\vee}\right) .
$$

Denote by $\mathscr{Y}_{\chi} \subset H_{n} \times_{k} B \times_{k} \mathbb{P}(V)$ the pullback under $\chi \times \operatorname{Id}_{\mathbb{P}(V)}$ of the universal degree $d$ hypersurface $9 \subset \mathbb{P} \operatorname{Sym}^{d}\left(V^{\vee}\right) \times_{k} \mathbb{P}(V)$. For every field $k^{\prime}$ and every $[j] \in H_{n}\left(k^{\prime}\right)$, denote by $\mathscr{Y}_{j}$ the restriction of $\mathscr{Y}_{\chi}$ to $\operatorname{Spec}\left(k^{\prime}\right) \times B$. Also denote by $K^{\prime}$ the function field $k^{\prime}(B)$, and denote by $\mathscr{y}_{j, K^{\prime}}$ the fiber of $\mathscr{y}_{j}$ over the generic point $\operatorname{Spec}\left(K^{\prime}\right)$ of $k^{\prime}(B)$.

Corollary 2.3. Assume $d>n$. In $H_{n}$ there is a countable intersection of open dense subsets such that for every $[j]$ in this set, $M\left(K^{\prime}, \mathscr{Y}_{j, K^{\prime}}\right)=d$ and $I\left(K^{\prime}, \mathscr{Y}_{j, K^{\prime}}\right)$ is divisible by $\operatorname{gcd}\left(d, \ldots,\left(\begin{array}{l}d \\ n\end{array}\right)\right)$. In particular this holds when $[j]$ equals the geometric generic point of $H_{n}$.

Proof. The subset $H_{n}^{\text {good }} \subset H_{n}$ where $\operatorname{gcd}\left(d, \ldots,\left(\begin{array}{l}d \\ n\end{array}\right)\right)$ divides $I\left(K^{\prime}, \mathscr{X}_{h, K^{\prime}}\right)$ and where $M\left(K^{\prime}, \mathscr{X}_{K^{\prime}}\right)$ is at least $d$ is a countable intersection of open subsets by standard Hilbert scheme arguments: the complement of this set is the union indexed by the countably many Hilbert polynomials $P(t)$ of multisections of degree which are either less than $d$ or which are not divisible by $\operatorname{gcd}\left(d, \ldots,\left(\begin{array}{l}d \\ n\end{array}\right)\right)$ of the closed image in $H_{n}$ of the relative Hilbert scheme $\operatorname{Hilb}_{\mathscr{L} / H_{n}}^{P(t)}$. By Proposition $2.2 H_{n}^{\text {good }}$ is nonempty. Therefore it is a countable intersection of open dense subsets. Of course 
the intersection of $\mathscr{Y}_{h, K^{\prime}}$ with a general line in $\mathbb{P}\left(V \otimes_{k} K^{\prime}\right)$ gives a multisection of degree $d$. Therefore $H_{n}^{\text {good }}$ is actually the set where $M\left(K^{\prime}, \mathscr{X}_{K^{\prime}}\right)$ equals $d$ and where $\operatorname{gcd}\left(d, \ldots,\left(\begin{array}{l}d \\ n\end{array}\right)\right)$ divides $I\left(K^{\prime}, \mathscr{X}_{h, K^{\prime}}\right)$.

2.1. Proof of Proposition 1.2. Let $k$ be an algebraically closed field having infinite transcendence degree over its prime subfield. The main case of Proposition 1.2 is actually the special case where $B$ equals $\mathbb{P}_{k}^{1} \times_{k} \mathbb{P}_{k}^{1}$ and where the family of curves $(M, \mathscr{C})$ is the complete linear system $|\mathcal{O}(a, b)|$ for some integers $a, b \geq 0$.

Assume first that one of $a$ or $b$ equals 0 , say $b=0$. Let $f: Y \rightarrow \mathbb{P}_{k}^{1}$ be a finite, separably-generated morphism of irreducible curves of degree $>1$, and let $\mathscr{X}$ be $Y \times \mathbb{P}^{1}$ with projection $\pi=(f$, Id). Every divisor in $|\mathcal{O}(a, 0)|$ is a union of fibers of $\mathrm{pr}_{1}$, so the restriction of $\pi$ has a section. The restriction of $\pi$ over every fiber of $\mathrm{pr}_{2}$ is just $f$, and so has no rational section.

Thus assume next that both $a$ and $b$ are positive. Define $n=4 a b$ and define $d=n-1$. Let $V$ be a $k$-vector space of dimension $n+1$ as above. Let $C \subset \mathbb{P}^{1} \times \mathbb{P}^{1}$ be a smooth curve in the linear system $|\mathcal{O}(1,2 b)|$. By Corollary 2.3, there exists a closed immersion of degree $n, j: C \rightarrow \mathbb{P} \operatorname{Sym}^{d}\left(V^{\vee}\right)$, such that $M\left(k(C), \mathscr{Y}_{j, k(C)}\right)$ equals $d$, which is $>1$. Of course $j$ extends to a closed immersion

$$
j: \mathbb{P}^{1} \times \mathbb{P}^{1} \rightarrow \mathbb{P} \operatorname{Sym}^{d}\left(V^{\vee}\right)
$$

such that $j^{*} O(1)$ is $O(2 a-1,2 b)$. Indeed the restriction map

$$
H^{0}\left(\mathbb{P}^{1} \times \mathbb{P}^{1}, \mathcal{O}(2 a-1,2 b)\right) \rightarrow H^{0}\left(C, \mathscr{O}_{C}(n)\right)
$$

is surjective. Define $\pi: \mathscr{L} \rightarrow \mathbb{P}^{1} \times \mathbb{P}^{1}$ to be the pullback under $j \times \operatorname{Id}_{\mathbb{P}(V)}$ of the universal hypersurface $9 y \subset \mathbb{P} \operatorname{Sym}^{d}\left(V^{\vee}\right) \times_{k} \mathbb{P}(V)$. By construction, the restriction over $C$ has no section.

Every divisor in $|\mathcal{O}(a, b)|$ maps under $j$ to a curve in $\mathbb{P} \operatorname{Sym}^{d}\left(V^{\vee}\right)$ whose degree with respect to $O(1)$ is $\leq n-b$. Moreover, if the degree equals $n-b$, then $j$ maps the divisor birationally to its image. Thus the arithmetic genus $p_{a}$ of the image is at least the geometric genus of the divisor, that is, $p_{a} \geq(a-1)(b-1)$. A curve of arithmetic genus $p_{a}$ and degree $\delta$ spans a linear space of (projective) dimension $\leq \delta-p_{a}$. Thus the span of the image of the divisor is either $\leq n-b-1$ (if $\delta \leq n-b-1)$ or $\leq n-b-(a-1)(b-1)$ which is again $\leq n-b-1$ (when $\delta$ equals $n-b)$. The span is a linear system of hypersurfaces in $\mathbb{P}(V)$. Since $n-b-1$ is $\leq n$, this linear system has a nonempty base locus. But every point in the base locus gives a section of the corresponding family of hypersurfaces. Thus it also gives a section of the restriction of $\mathscr{X}$ over the divisor. This proves the main case of Proposition 1.2, that is, the case when $B$ equals $\mathbb{P}^{1} \times \mathbb{P}^{1}$ and when $M$ is a complete linear system $|\mathcal{O}(a, b)|$. 
Next let $B$ be an arbitrary normal, projective variety of dimension $\geq 2$ and let $M$ be an irreducible family of irreducible curves dominating $B$. There exists a smooth open subset $U \subset B$ whose complement has codimension $\geq 2$, and there exists a dominant morphism $g: U \rightarrow \mathbb{P}^{1} \times \mathbb{P}^{1}$. Intersecting $U$ with general hyperplanes, there exists an irreducible closed subset $Z \subset U$ such that $\left.g\right|_{Z}: Z \rightarrow \mathbb{P}^{1} \times \mathbb{P}^{1}$ is generically finite of some degree $e>0$. For the geometric generic point of $M$, the intersection of the corresponding curve with $U$ is nonempty, and the closure of the image under $f$ is a divisor in the linear system $\left|\mathcal{O}\left(a^{\prime}, b^{\prime}\right)\right|$ for some nonnegative integers $a^{\prime}, b^{\prime}$. Let $a \geq a^{\prime}$, and $b \geq b^{\prime}$ be integers such that $4 a b>e+1$. There exists a projective, dominant morphism $\pi: \mathscr{X} \rightarrow \mathbb{P}^{1} \times \mathbb{P}^{1}$ whose restriction over every divisor in $|\mathcal{O}(a, b)|$ has a section, but whose restriction over a general divisor in $|\mathcal{O}(1,2 b)|$ has minimal degree $4 a b-1$.

Define $\mathscr{X}_{B} \subset B \times \mathscr{X}$ to be the closure of $U \times_{\mathbb{P}^{1} \times \mathbb{P}^{1}} \mathscr{X}$. Then $\pi_{B}: \mathscr{X}_{B} \rightarrow B$ is a projective dominant morphism. For the geometric generic point of $M$, the restriction of $\pi_{B}$ to the curve has a section because the restriction of $\pi$ to the image in $\mathbb{P}^{1} \times \mathbb{P}^{1}$ has a section. Let $C_{B} \subset Z$ be the preimage of a general curve $C$ in $|\mathcal{O}(1,2 b)|$. The morphism $C_{B} \rightarrow C$ has degree $e<4 a b-1$. Because every multisection of $\pi$ over $C$ has degree $\geq 4 a b-1, \pi_{B}$ has no section over $C_{B}$.

\section{The construction for Enriques surfaces}

Next let $k$ be a field of characteristic $\neq 2,3$ whose transcendence degree over the prime subfield is "sufficiently large". In fact, let us simply assume that $k$ is uncountable. It is straightforward to trace through the following arguments to find an integer $N$ such that everything remains valid if the transcendence degree over the prime subfield is $\geq N$. However, we think this extra bookkeeping would only distract from the proof, which is already burdened by heavy notation.

We will make use of one particular construction of Enriques surfaces over $k$. To that end, let $V_{+}$and $V_{-}$be three-dimensional $k$-vector spaces. Denote $V=$ $V_{+} \oplus V_{-}$and denote $V^{\prime}=\operatorname{Sym}^{2}\left(V_{+}^{\vee}\right) \oplus \operatorname{Sym}^{2}\left(V_{-}^{\vee}\right)$. Denote by $G$ the Grassmannian $\operatorname{Grass}\left(3, V^{\prime}\right)$ parametrizing three-dimensional subspaces of $V^{\prime}$. This is a parameter space for Enriques surfaces, as we shall explain.

In fact there are two descriptions of the universal family, each useful. First, let $\pi_{Z}: Z \rightarrow \mathbb{P}\left(V_{+}\right) \times \mathbb{P}\left(V_{-}\right)$be the projective bundle of the locally free sheaf pr $_{+}^{*} \mathcal{O}_{\mathbb{P}\left(V_{+}\right)}(-2) \oplus \mathrm{pr}_{-}^{*} \mathbb{O}_{\mathbb{P}\left(V_{-}\right)}(-2)$. A general complete intersection of three divisors in $\left|O_{Z}(1)\right|$ is an Enriques surface. Because $H^{0}\left(Z, O_{Z}(1)\right)=V^{\prime}$, the parameter space for these complete intersections is $G$. Second, $G$ parametrizes complete intersections in $\mathbb{P}(V)$ of three quadric divisors that are invariant under the involution $\imath$ of $\mathbb{P}(V)$ whose $(-1)$-eigenspace is $V_{-}$and whose $(+1)$-eigenspace is $V_{+}$. A general such complete intersection is a K3 surface on which $l$ acts as a fixed-pointfree involution. The quotient by $\imath$ is an Enriques surface. 
These two descriptions are equivalent. The involution extends to an involution $\tilde{l}$ on the blowing up $\widetilde{\mathbb{P}(V)}$ of $\mathbb{P}(V)$ along $\mathbb{P}\left(V_{+}\right) \cup \mathbb{P}\left(V_{-}\right)$and the quotient is $Z$. Denote by $\mathscr{X} \rightarrow G$ the universal family of Enriques surfaces, and denote by $\mathscr{Y} \rightarrow G$ the universal family of $\mathrm{K} 3$ covers.

As in the proof of Corollary 2.3, the one-parameter family of Enriques surfaces in Theorem 1.1 will be a general deformation of a one-parameter family of reducible surfaces. As in Proposition 2.2, the one-parameter family of reducible surfaces will be constructed using a particular cover of $\mathbb{P}^{1}$ by $\mathbb{P}^{1}$. Thus let $B, C, D$ be $k$-curves isomorphic to $\mathbb{P}_{k}^{1}$. By a result similar to Lemma 2.1 , there exists a degree 2, separably-generated morphism $g: D \rightarrow C$ and a degree 3, separablygenerated morphism $f: C \rightarrow B$ such that $\operatorname{Gal}(k(D) / k(B))$ is the full wreath product $\mathfrak{W}_{3,2}$, that is, the semidirect product $\left(\mathfrak{S}_{2}\right)^{3} \rtimes \mathfrak{S}_{3}$. In characteristic 0 , this holds whenever $g$ and $f$ have simple branching and the branch points of $g$ are in distinct, reduced fibers of $f$. There is an involution $\imath_{D}$ of $D$ commuting with $g$.

Now we will construct the family of reducible surfaces using norms as in Section 2. Let $j: D \rightarrow \mathbb{P}\left(V^{\vee}\right)$ be a closed immersion equivariant for $\iota_{D}$ and $l$ whose image is a rational normal curve of degree 5. By the construction in Section 2, there is an associated morphism $i: C \rightarrow \mathbb{P} \operatorname{Sym}^{2}\left(V^{\vee}\right)$. Because $j$ is equivariant, $i$ factors through $\mathbb{P}\left(V^{\prime}\right)$. In the rest of this paragraph we will compute that

$$
i^{*} \mathcal{O}(1)=\operatorname{Nm}_{g}\left(j^{*} \mathcal{O}(1)\right) \cong \mathscr{O}_{C}(5) .
$$

Indeed, $j^{*} O(1)$ has degree 5 on $D$ by construction. And $g$ has degree 2. Thus $j^{*} \mathcal{O}(1) \otimes g^{*} \mathscr{O}_{C}(-3)$ has degree -1 , hence has both $h^{0}=0$ and $h^{1}=0$. Since $g$ is affine, $g_{*}$ is exact on $\mathrm{O}_{D}$-modules and $R^{i} g_{*}$ is zero on $\mathrm{O}_{D}$-modules for $i>0$. Thus, by a Leray spectral sequence, $g_{*} j^{*} \mathcal{O}(1) \otimes 0_{C} \mathscr{O}_{C}(-3)$ is a rank 2 locally free sheaf with $h^{0}=0$ and $h^{1}=0$. By Grothendieck's lemma, this sheaf is a direct sum of two invertible sheaves. And the condition on $h^{0}$ and $h^{1}$ implies that $g_{*} j^{*} \mathcal{O}(1) \otimes \mathscr{O}_{C} \mathcal{O}_{C}(-3)$ is isomorphic to $\mathscr{O}_{c}(-1)^{\oplus 2}$. Thus $g_{*} j^{*} \mathcal{O}(1)$ is isomorphic to $\bigcirc_{C}(2)^{\oplus 2}$, which has determinant $\bigcirc_{C}(4)$. Again by considering $h^{0}$ and $h^{1}$ and using Grothendieck's lemma, $g_{*} \hat{O}_{D}$ is isomorphic to $\mathscr{O}_{C} \oplus \mathscr{O}_{C}(-1)$, which has determinant $\mathscr{O}_{C}(-1)$. Thus $\mathrm{Nm}_{g}\left(j^{*} \mathcal{O}(1)\right)$ is isomorphic to $\operatorname{Hom}_{\mathscr{O}_{C}}\left(\mathscr{O}_{C}(-1), \mathscr{O}_{C}(4)\right)$, i.e., $\mathscr{O}_{C}(5)$.

The pushforward by $f_{*}$ of the pullback by $i^{*}$ of the tautological surjection is a surjection $\left(V^{\prime}\right)^{\vee} \otimes \mathcal{O}_{B} \rightarrow f_{*} i^{*} \mathcal{O}(1)$. The sheaf $f_{*} i^{*} \mathcal{O}(1)$ is locally free of rank 3 . By the same type of analysis of $h^{0}$ and $h^{1}$ as in the previous paragraph, $f_{*} \mathscr{O}_{C}(5) \otimes_{O_{B}}$ $\mathrm{O}_{B}(-2)$ is isomorphic to $\mathscr{O}_{B}(-1)^{\oplus 3}$. Thus $f_{*} i^{*} \mathcal{O}(1)$, that is, $f_{*} i^{*} \mathscr{O}_{C}(5)$, is isomorphic to $\mathrm{O}_{B}(1)^{3}$. So there is an induced morphism $h: B \rightarrow G$. Denote by $\pi_{h}: \mathscr{X}_{h} \rightarrow B$ and $\rho_{h}: \mathscr{Y}_{h} \rightarrow B$ the base-change by $h$ of $\mathscr{X}$ and $\mathscr{Y}$. Denote $K=k(B)$ and denote by $\mathscr{X}_{h, K}$ the generic fiber of $\pi_{h}$.

Proposition 3.1. Every irreducible multisection of $\pi_{h}$ has degree divisible by 3 or 4. In particular $M\left(K, \mathscr{X}_{h, K}\right)=3$. 
Proof. This is a combinatorial analysis, of precisely the sort we avoided in the proof of Lemma 2.1. Unfortunately here it seems necessary. Denote by $U \subset B$ the open set over which $f \circ g$ is étale, and denote by $W \subset D$ the preimage of $U$. Denote by $c: \widetilde{W} \rightarrow U$ the Galois closure of $W / U$. Then $\left.c^{*} f_{*} \mathcal{O}_{C}\right|_{U} \cong \mathcal{O}_{\widetilde{W}}\left\{\boldsymbol{a}_{1}, \boldsymbol{a}_{2}, \boldsymbol{a}_{3}\right\}$ for idempotents $\boldsymbol{a}_{p}, p=1,2,3$. And $\left.c^{*} g_{*} f_{*} \mathcal{O}_{D}\right|_{U} \cong \mathbb{O}_{\widetilde{W}}\left\{\boldsymbol{b}_{1,1}, \boldsymbol{b}_{1,2}, \boldsymbol{b}_{2,1}, \boldsymbol{b}_{2,2}, \boldsymbol{b}_{3,1}, \boldsymbol{b}_{3,2}\right\}$ for idempotents $\boldsymbol{b}_{p, q}, p=1,2,3, q=1,2$. Of course $\boldsymbol{a}_{p} \mapsto \boldsymbol{b}_{p, 1}+\boldsymbol{b}_{p, 2}, p=1,2,3$. The action of the Galois group $\mathfrak{W}_{3,2}$ on $\boldsymbol{a}_{p}$ is by the symmetric group $\mathfrak{S}_{3}$, and the action on $\boldsymbol{b}_{p, q}$ is the standard representation of the wreath product. In the next paragraph, every index $p$ corresponds to an index $p$ of $\boldsymbol{a}_{p}$. As here, these induces are permuted by $\mathfrak{W}_{3,2}$ through its quotient $\mathfrak{S}_{3}$. And in the next paragraph, every index $(p, q)$ corresponds to an index $(p, q)$ of $\boldsymbol{b}_{p, q}$. The action of $\mathfrak{W}_{3,2}$ on these indices is the standard representation of the wreath product.

For each $p=1,2,3$ and $q=1,2$, denote by $j_{p, q}: \widetilde{W} \rightarrow \mathbb{P}\left(V^{\vee}\right)$ the morphism obtained by composing the idempotent $\boldsymbol{b}_{p, q}: \widetilde{W} \rightarrow \widetilde{W} \times_{U} W$ with the basechange of $j$. In particular, $\iota \circ j_{p, 1}=j_{p, 2}$. Denote by $\Lambda_{p, q} \subset \widetilde{W} \times \mathbb{P}(V)$ the pullback by $\left(j_{p, q}\right.$, Id) of the universal hyperplane. Denote by $\mathscr{Y}_{\widetilde{W}}$ the base-change to $\widetilde{W}$ of $\mathscr{Y}_{h}$. Then

$$
\mathscr{Y}_{\widetilde{W}}=\bigcup_{\left(q_{1}, q_{2}, q_{3}\right) \in\{1,2\}^{3}}\left(\Lambda_{1, q_{1}} \cap \Lambda_{2, q_{2}} \cap \Lambda_{3, q_{3}}\right) .
$$

There is a locally closed stratification

$$
y_{\widetilde{W}}=\mathscr{Y Y}_{\widetilde{W}}^{3} \sqcup \mathscr{Y}_{\widetilde{W}}^{4} \sqcup \mathscr{Y}_{\widetilde{W}}^{5},
$$

where $y_{\widetilde{W}}^{l}$ is the set of points lying in the intersection of precisely $l$ of the $\Lambda_{p, q}$. The stratum $\mathcal{Y}_{\widetilde{W}}^{3}$ is the union of eight connected open subsets,

$$
\Lambda_{\left(q_{1}, q_{2}, q_{3}\right)} \subset\left(\Lambda_{1, q_{1}} \cap \Lambda_{2, q_{2}} \cap \Lambda_{3, q_{3}}\right),
$$

for $q_{1}, q_{2}, q_{3} \in\{1,2\}$. Each connected component is a dense open subset of a $\mathbb{P}^{2}$-bundle over $\widetilde{W}$. The stratum $\mathcal{Y}_{\widetilde{W}}^{4}$ is the union of 12 connected open subsets,

$$
\begin{aligned}
& \Lambda_{\left(*, q_{2}, q_{3}\right)} \subset\left(\Lambda_{1,1} \cap \Lambda_{1,2}\right) \cap \Lambda_{2, q_{2}} \cap \Lambda_{3, q_{3},}, \\
& \Lambda_{\left(q_{1}, *, q_{3}\right)} \subset \Lambda_{1, q_{1}} \cap\left(\Lambda_{2,1} \cap \Lambda_{2,2}\right) \cap \Lambda_{3, q_{3}}, \\
& \Lambda_{\left(q_{1}, q_{2}, *\right)} \subset \Lambda_{1, q_{1}} \cap \Lambda_{2, q_{2}} \cap\left(\Lambda_{3,1} \cap \Lambda_{3,2}\right),
\end{aligned}
$$

for $q_{1}, q_{2}, q_{3} \in\{1,2\}$. Each connected component is a dense open subset of a $\mathbb{P}^{1}$-bundle over $\widetilde{W}$. Finally $y_{\widetilde{W}}^{5}$ is the union of the six connected sets

$$
\begin{aligned}
& \Lambda_{\left(*, *, q_{3}\right)}=\left(\Lambda_{1,1} \cap \Lambda_{1,2}\right) \cap\left(\Lambda_{2,1} \cap \Lambda_{2,2}\right) \cap \Lambda_{3, q_{3}}, \\
& \Lambda_{\left(*, q_{2}, *\right)}=\left(\Lambda_{1,1} \cap \Lambda_{1,2}\right) \cap \Lambda_{2, q_{2}} \cap\left(\Lambda_{3,1} \cap \Lambda_{3,2}\right), \\
& \Lambda_{\left(q_{1}, *, *\right)}=\Lambda_{1, q_{1}} \cap\left(\Lambda_{2,1} \cap \Lambda_{2,2}\right) \cap\left(\Lambda_{3,1} \cap \Lambda_{3,2}\right),
\end{aligned}
$$

for $q_{1}, q_{2}, q_{3} \in\{1,2\}$. Each connected component projects isomorphically to $\widetilde{W}$. 
There is a bijection between multisections of $\mathscr{Y}_{h}$ over $U$ and Galois invariant multisections of $\mathscr{Y}_{\widetilde{W}}$ over $\widetilde{W}$. An irreducible multisection of $\mathscr{Y}_{h}$ determines a multisection of $\mathscr{Y}_{\widetilde{W}}$ contained in a single stratum $\mathscr{Y}_{\widetilde{W}}^{l}$. The action of the Galois group $\mathfrak{W}_{3,2}$ on the connected components of $\mathscr{y}_{\widetilde{W}}^{l}$ is the obvious one; in particular, it acts transitively on the set of connected components. So every Galois invariant multisection in $\mathcal{Y}_{\widetilde{W}}^{3}$ has degree divisible by 8 , every Galois invariant multisection in $\mathscr{Y}_{\widetilde{W}}^{4}$ has degree divisible by 12 , and every Galois invariant multisection in $\mathscr{Y}_{\widetilde{W}}^{5}$ has degree divisible by 6 . Therefore every irreducible multisection of $\mathscr{Y}_{h}$ has degree divisible by 8 or 6 . Because $\mathscr{Y}_{h}$ is a double-cover of $\mathscr{L}_{h}$, every irreducible multisection of $\mathscr{X}_{h}$ has degree divisible by 4 or 3 . In particular, the minimal degree of a multisection of $\mathscr{X}_{h}$ is 3 .

Because $f_{*} i^{*} \mathscr{O}(1) \cong \mathscr{O}_{B}(1)^{3}$, the scheme $\mathscr{L}_{h} \subset B \times Z$ is a complete intersection of three divisors in the linear system $\left|\operatorname{pr}_{B}^{*} O_{B}(1) \otimes p_{Z}^{*} O_{Z}(1)\right|$. A general deformation of this complete intersection is a pencil of Enriques surfaces satisfying Theorem 1.1 (i) and (ii) with $M\left(K, X_{K}\right) \geq 3, I\left(K, X_{K}\right) \mid 4$ (this is valid so long as $\operatorname{char}(k) \neq 2$ ). For (iii), it is necessary to deform the pencil together with the degree 3 multisection. This requires the hypothesis that $\operatorname{char}(k) \neq 2,3$. We explain the argument below.

The stratum $\mathscr{Y}_{\widetilde{W}}^{5}$ is Galois invariant and determines a degree 3 multisection of $\mathscr{X}_{h}$. As a $\mathfrak{W}_{3,2}$-equivariant morphism to $\widetilde{W}, \mathscr{Y}_{\widetilde{W}}^{5}$ is just the base-change of $D$, and the morphism $\mathcal{Y}_{\widetilde{W}}^{5} \rightarrow \mathbb{P}(V)$ is Galois invariant. By étale descent it is the basechange of a morphism $j^{\prime}: D \rightarrow \mathbb{P}(V)$. Now $j^{\prime}$ induces a morphism to $\widetilde{\mathbb{P}(V)}$, the blowing up of $\mathbb{P}(V)$ along $\mathbb{P}\left(V_{+}\right) \cup \mathbb{P}\left(V_{-}\right)$. Because $j^{\prime}$ is equivariant for $\imath$ and $\imath_{D}$, the quotient morphism $D \rightarrow Z$ factors through $C$, that is, there is an induced morphism $i^{\prime}: C \rightarrow Z$. By a straightforward enumerative geometry computation, $j^{\prime}$ has degree 5 with respect to $\mathbb{O}_{\mathbb{P}(V)}(1)$. Therefore $i^{\prime}$ has degree 5 with respect to $\mathrm{O}_{Z}(1)$. The degree 3 multisection of $\mathscr{X}_{h}$ is the image of $\left(f, i^{\prime}\right): C \rightarrow B \times Z$.

Lemma 3.2. If $f, g$ and $j$ are general, then $\left(i^{\prime}\right)^{*}: H^{0}\left(Z, O_{Z}(1)\right) \rightarrow H^{0}\left(C, O_{C}(5)\right)$ is surjective.

Proof. The condition that $\left(i^{\prime}\right)^{*}$ is surjective is an open condition in families, hence it suffices to verify $\left(i^{\prime}\right)^{*}$ is surjective for a single choice of $f, g$ and $j$, even one for which $\operatorname{Gal}(k(D) / k(B))$ is not $\mathfrak{W}_{3,2}$. Choose homogeneous coordinates $\left[S_{0}, S_{1}\right]$ on $D,\left[T_{0}, T_{1}\right]$ on $C$ and $\left[U_{0}, U_{1}\right]$ on $B$. Define $g\left(\left[S_{0}, S_{1}\right]\right)=\left[S_{0}^{2}, S_{1}^{2}\right]$ and $f\left(\left[T_{0}, T_{1}\right]\right)=\left[T_{0}^{3}, T_{1}^{3}\right]$. Denote by $\mu_{6}$ the group scheme of 6 th roots of unity. There is an action of $\mu_{6}$ on $D$ by $\zeta \cdot\left[S_{0}, S_{1}\right]=\left[S_{0}, \zeta S_{1}\right]$. This identifies $\mu_{6}$ with $\operatorname{Gal}(k(D) / k(B))$.

Let $\boldsymbol{e}_{+, 0}, \boldsymbol{e}_{+, 1}, \boldsymbol{e}_{+, 2}$ and $\boldsymbol{e}_{-, 0}, \boldsymbol{e}_{-, 1}, \boldsymbol{e}_{-, 2}$ be ordered bases of $V_{+}$and $V_{-}$respectively, and let $X_{+, 0}, X_{+, 1}, X_{+, 2}$ and $X_{-, 0}, X_{-, 1}, X_{-, 2}$ be the dual ordered bases of $V_{+}^{\vee}$ and $V_{-}^{\vee}$ respectively. There is an action of $\mu_{6}$ on $V$ by 
$\zeta \cdot\left[X_{+, 0}, X_{+, 1}, X_{+, 2}, X_{-, 0}, X_{-, 1}, X_{-, 2}\right]$

$$
=\left[X_{+, 0}, \zeta^{2} X_{+, 1}, \zeta^{4} X_{+, 2}, \zeta X_{-, 0}, \zeta^{3} X_{-, 1}, \zeta^{5} X_{-, 2}\right]
$$

and a dual action on $V^{\vee}$. Define $j: D \rightarrow \mathbb{P}(V)$ with respect to the ordered basis $\mathbf{e}_{+, 0}, \ldots, \mathbf{e}_{-, 2}$ to be the $\mu_{6}$-equivariant morphism

$$
j\left(\left[S_{0}, S_{1}\right]\right)=\left[S_{0}^{5}, S_{0}^{3} S_{1}^{2}, S_{0} S_{1}^{3}, S_{0}^{4} S_{1}, S_{0}^{2} S_{1}^{3}, S_{1}^{5}\right] .
$$

In this case $U=D_{+}\left(U_{0} U_{1}\right) \subset B$ and $\widetilde{W}=W=D_{+}\left(S_{0} S_{1}\right) \subset C$. It is straightforward to compute $j^{\prime}$ with respect to the dual ordered basis $X_{+, 0}, \ldots, X_{-, 2}$ :

$$
j^{\prime}\left(\left[S_{0}, S_{1}\right]\right)=\left[S_{1}^{5}, S_{0}^{2} S_{1}^{3}, S_{0}^{4} S_{1}, S_{0} S_{1}^{4}, S_{0} S_{1}^{4}, S_{0}^{3} S_{1}^{2}, S_{0}^{5}\right] .
$$

As a double-check, observe this is $\mu_{6}$-equivariant. The induced map $\left(j^{\prime}\right)^{*}$ is

$$
\begin{array}{lll}
X_{+, 0} X_{+, 0} \mapsto T_{1}^{5}, & X_{+, 0} X_{+, 1} \mapsto T_{0} T_{1}^{4}, & X_{+, 0} X_{+, 2} \mapsto T_{0}^{2} T_{1}^{3}, \\
X_{+, 1} X_{+, 1} \mapsto T_{0}^{2} T_{1}^{3}, & X_{+, 1} X_{+, 2} \mapsto T_{0}^{3} T_{1}^{2}, & X_{+, 2} X_{+, 2} \mapsto T_{0}^{4} T_{1}, \\
X_{-, 0} X_{-, 0} \mapsto T_{0} T_{1}^{4}, & X_{-, 0} X_{-, 1} \mapsto T_{0}^{2} T_{1}^{3}, & X_{-, 0} X_{-, 2} \mapsto T_{0}^{3} T_{1}^{2}, \\
X_{-, 1} X_{-, 1} \mapsto T_{0}^{3} T_{1}^{2}, & X_{-, 1} X_{-, 2} \mapsto T_{0}^{4} T_{1}, & X_{-, 2} X_{-, 2} \mapsto T_{0}^{5} .
\end{array}
$$

This is surjective by inspection.

Proof of Theorem 1.1. The subvariety $\mathscr{L}_{h} \subset B \times Z$ is a complete intersection of three divisors in the linear system $\left|\operatorname{pr}_{B}^{*} O_{B}(1) \otimes \operatorname{pr}_{Z}^{*} O_{Z}(1)\right|$, each containing $\left(f, i^{\prime}\right)(C)$. Denote by $\mathscr{I}$ the ideal sheaf of $\left(f, i^{\prime}\right)(C) \subset B \times Z$, and set

$$
I=H^{0}\left(B \times Z, \mathscr{I} \otimes \operatorname{pr}_{B}^{*} O_{B}(1) \otimes \operatorname{pr}_{Z}^{*} O_{Z}(1)\right) .
$$

The projective space of $I$ is the linear system of divisors on $B \times Z$ in the linear system $\left|\operatorname{pr}_{B}^{*} O_{B}(1) \otimes \operatorname{pr}_{Z}^{*} O_{Z}(1)\right|$ that contain $\left(f, i^{\prime}\right)(C)$. The Grassmannian $G^{\prime}=$ $\operatorname{Grass}(3, I)$ is the parameter space for deformations of $\mathscr{X}_{h}$ that contain $\left(f, i^{\prime}\right)(C)$. For the same reason as in Corollary 2.3, in $G^{\prime}$ there is a countable intersection of dense open subsets parametrizing subvarieties $\mathscr{X}^{\prime} \subset B \times Z$ with $M\left(K, \mathscr{X}_{K}^{\prime}\right) \geq 3$ and $I\left(K, \mathscr{X}_{K}^{\prime}\right) \mid 4$. By construction, $\mathscr{L}^{\prime}$ contains the degree 3 multisection $\left(f, i^{\prime}\right)(C)$. Therefore $M\left(K, \mathscr{X}_{K}^{\prime}\right)=3$ and $I\left(K, \mathscr{X}_{K}^{\prime}\right)=1$. It is straightforward to compute $\operatorname{pr}_{B} *\left[\omega_{\mathscr{X}^{\prime} / B}^{\otimes 2}\right] \cong \mathcal{O}_{B}(6)$. So to prove the theorem, it suffices to prove every "very general" Enriques surface occurs as a fiber of some $\mathscr{X}^{\prime}$, that is, for a general $[X] \in G$, $X$ occurs as $\operatorname{pr}_{Z}\left(\mathscr{C}^{\prime} \cap \pi_{B}^{-1}(b)\right)$ for some choice of $f, g, i$ and $b \in B$.

A general zero-dimensional, length 3 subscheme of $Z$ occurs as $i^{\prime}\left(f^{-1}(b)\right)$ for some choice of $f, g, i$ and $b \in B$. So for a general Enriques surface $[X] \in G$ and a general choice of zero-dimensional, length 3 subscheme of $X, X$ is a complete intersection of three divisors in the linear system $\left|O_{Z}(1)\right|$ containing $i^{\prime}\left(f^{-1}(b)\right)$ for some choice of $f, g, i$ and $b$. To prove that a general $[X] \in G$ is the fiber over $b$ of $\mathscr{X}^{\prime}$ for some $f, g, i$ and $\left[\mathscr{X}^{\prime}\right] \in G^{\prime}$, it suffices to prove every divisor in the linear 
system $\left|O_{Z}(1)\right|$ containing $i^{\prime}\left(f^{-1}(b)\right)$ is the fiber over $b$ of a divisor in the linear system $\left|\mathscr{T} \otimes O_{B}(1) \otimes O_{Z}(1)\right|$.

There is a short exact sequence

$$
\left.0 \rightarrow \mathscr{I} \otimes \operatorname{pr}_{Z}^{*} \mathrm{O}_{Z}(1) \rightarrow \operatorname{pr}_{Z}^{*} \mathrm{O}_{Z}(1) \rightarrow \operatorname{pr}_{Z}^{*} \mathrm{O}_{Z}(1)\right|_{C} \rightarrow 0
$$

giving a short exact sequence

$$
0 \rightarrow \operatorname{pr}_{B, *}\left(\mathscr{I} \otimes \operatorname{pr}_{Z}^{*} \mathrm{O}_{Z}(1)\right) \rightarrow \operatorname{pr}_{B, *} \operatorname{pr}_{Z}^{*} O_{Z}(1) \rightarrow \operatorname{pr}_{B, *}\left(\left.\operatorname{pr}_{Z}^{*} O_{Z}(1)\right|_{C}\right) \rightarrow 0 .
$$

Because $\left(i^{\prime}\right)^{*}$ is surjective, $\operatorname{pr}_{B, *}\left(\mathscr{I} \otimes \operatorname{pr}_{Z}^{*} O_{Z}(1)\right)$ is a locally free sheaf with $h^{1}=0$.

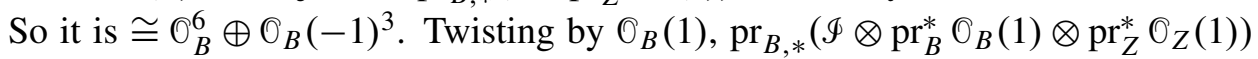
is generated by global sections. Therefore every divisor on $Z$ in the linear system $\left|O_{Z}(1)\right|$ containing the scheme $i^{\prime}\left(f^{-1}(b)\right)$ is the fiber over $b$ of a divisor on $B \times Z$ in the linear system $\left|\mathscr{I} \otimes \operatorname{pr}_{B}^{*} O_{B}(1) \otimes \operatorname{pr}_{Z}^{*} O_{Z}(1)\right|$.

\section{Acknowledgments}

I am grateful to Igor Dolgachev, Hélène Esnault, Mike Stillman and Harry Tamvakis for useful conversations. This paper originated in meetings with Tom Graber, Joe Harris and Barry Mazur to whom I am very grateful. I am also grateful to the referee for many useful suggestions.

\section{References}

[Colmez and Serre 2001] P. Colmez and J.-P. Serre (editors), Correspondance Grothendieck-Serre, Documents Mathématiques 2, Société Mathématique de France, Paris, 2001. MR 2003k:14002

[Deligne and Katz 1973] P. Deligne and N. Katz (editors), Groupes de monodromie en géométrie algébrique, II: Séminaire de Géométrie Algébrique du Bois-Marie 1967-1969 (SGA 7 II), Lecture Notes in Math. 340, Springer, Berlin, 1973. MR 50 \#7135

[Graber et al. 2005] T. Graber, J. Harris, B. Mazur, and J. Starr, "Rational connectivity and sections of families over curves", Ann. Sci. École Norm. Sup. (4) 38:5 (2005), 671-692. MR 2006j:14044 Zbl 1092.14062

[Grothendieck 1961] A. Grothendieck, “Techniques de construction et théorèmes d'existence en géométrie algébrique, IV: Les schémas de Hilbert”, in Séminaire Bourbaki 1960/61 (Exposé 221), 1961. Reprinted as part IV in Fondements de la géométrie algébrique, Secrétariat mathématique, Paris, 1962. MR 26 \#3566 Zbl 0236.14003

[Grothendieck 1962] A. Grothendieck, Revêtements étales et groupe fondamental: Séminaire de géométrie algébrique du Bois Marie 1960-61 (SGA 1), Lecture Notes in Math. 224, Springer, Berlin, 1962. Reprinted Soc. math. de France, Paris, 2003. MR 50 \#7129

[Lafon 2004] G. Lafon, "Une surface d'Enriques sans point sur $\mathbb{C}((t))$ ", C. R. Math. Acad. Sci. Paris 338:1 (2004), 51-54. MR 2004k:14035 Zbl 1040.14020 
jstarr@math.sunysb.edu

Department of Mathematics, Stony Brook University, Stony Brook, NY 11790, United States http://www. math.sunysb.edu/ jstarr/ 